\title{
31P MR Spectroscopy with 3D Chemical-Shift Imaging Detects Changes in Levels of Phosphorus Metabolites Due to Saliva Secretion in Human Parotid Glands
}

\author{
Toshiyuki Sato*, Hiroyoshi Isoda, Kaori Togashi \\ Department of Diagnostic Imaging and Nuclear Medicine, Graduate School of Medicine, Kyoto University, \\ Shogoin-Kawaharacho, Sakyo-ku, Kyoto, Japan \\ Email: *yky50py@kuhp.kyoto-u.ac.jp, sayuki@kuhp.kyoto-u.ac.jp, ktogashi@kuhp.kyoto-u.ac.jp
}

How to cite this paper: Sato, T., Isoda, H. and Togashi, K. (2020) ${ }^{31} \mathrm{P}$ MR Spectroscopy with 3D Chemical-Shift Imaging Detects Changes in Levels of Phosphorus Metabolites Due to Saliva Secretion in Human Parotid Glands. Open Journal of Medical Imaging, 10, 42-51.

https://doi.org/10.4236ojmi.2020.101004

Received: January 17, 2020

Accepted: February 23, 2020

Published: February 26, 2020

Copyright $\odot 2020$ by author(s) and Scientific Research Publishing Inc. This work is licensed under the Creative Commons Attribution International License (CC BY 4.0).

http://creativecommons.org/licenses/by/4.0/

(c) (i) Open Access

\begin{abstract}
Objectives: To measure phosphorus metabolites in human parotid glands by ${ }^{31} \mathrm{P}$-MRS using three-dimensional chemical-shift imaging (3D-CSI), and ascertain whether this method can capture changes in adenosine triphosphate (ATP) and phosphocreatine (PCr) levels due to saliva secretion. Study Design: The parotid glands of 20 volunteers were assessed by ${ }^{31} \mathrm{P}$-MRS using 3D-CSI on 3T MRI. After obtaining a first (baseline) measurement, the participants took vitamin-C tablets and measurements were obtained twice more, in a continuous manner. The peak area ratios of $\mathrm{PCr}$ and $\beta$-ATP were evaluated. Results: A high proportion of PCr $(0.26 \pm 0.08)$ and ATP $(\alpha: 0.16$ $\pm 0.06 ; \beta: 0.27 \pm 0.06 ; \gamma .0 .21 \pm 0.03)$ was noted at baseline. A significant decrease in $\beta$-ATP was observed between baseline ("pre"; $0.27 \pm 0.06$ ) and the first scan after vitamin-C stimulation ("post-1"; $0.19 \pm 0.07, \mathrm{p}<0.05$ ). There was a near-significant decrease in PCr between pre $(0.26 \pm 0.08)$ and post-1 $(0.23 \pm 0.06, p=0.074)$. Conclusions: ${ }^{31} \mathrm{P}$-MRS with $3 \mathrm{D}$-CSI can assess the major phosphorus metabolites in human parotid glands and capture changes in their levels due to saliva secretion. This technique is simple, non-invasive, and provides new information regarding saliva secretion.
\end{abstract}

\section{Keywords}

${ }^{31}$ P-MRS, 3D-CSI, Parotid Gland

\section{Introduction}

The two parotid glands are the largest of the human salivary glands. They secrete 
saliva via the parotid duct into the oral cavity to facilitate mastication and swallowing. Resting (unstimulated) salivary production occurs mainly in the submandibular, sublingual, and numerous small oral salivary glands. In contrast, the parotid glands are largely responsible for stimulated salivary production ( $60 \%-70 \%$ of the total) [1]. Therefore, the change of function that occurs in the parotid glands at meal time is very important for appropriate dietary intake.

Magnetic resonance spectroscopy (MRS) classifies the magnetic-resonance signals of metabolites into frequencies using the difference in the resonance frequency of nuclei depending on the structure and environment of metabolites. MRS can be used to identify information such as the type of metabolite, its concentration, and the relaxation time. Phosphorus magnetic resonance spectroscopy $\left({ }^{31} \mathrm{P}\right.$-MRS) is a non-invasive technique that provides useful information regarding the metabolic and phosphoenergetic status of human tissue, in both physiological and pathological conditions [2] [3]. In addition to the information contained within static spectra, dynamic ${ }^{31} \mathrm{P}$-MRS has been used to assess changes in the levels of phosphorus metabolites [4] [5] [6]. Furthermore, three-dimensional chemical-shift imaging (3D-CSI) enables metabolic information and detailed information on position to be obtained [7] [8]. For the past three decades, ${ }^{31} \mathrm{P}-\mathrm{MRS}$ studies have been conducted using human tissues (e.g., muscle, heart, liver, brain); however, most studies on the salivary glands have been carried out in vitro in animal experiments [9]-[14], and only one study of static spectra in human parotid glands has been published [15].

Numerous previous studies have reported phosphocreatine ( $\mathrm{PCr}$ ) and adenosine triphosphate (ATP) levels measured by MRS in the excised glands of animals and in human parotid glands [9]-[15]. In addition, creatine phosphokinase has been purified from the submandibular glands of rats [16]. These findings indicate the existence of the Lohmann reaction in the salivary gland, in which adenosine triphosphate (ATP) is regenerated by transphosphorylation from $\mathrm{PCr}$ to adenosine diphosphate (ADP). It has been shown in MRS experiments using excised animal glands that ATP and PCr are consumed by salivary secretion [9]-[14], but reports on physiological changes in human salivary glands in vivo are lacking.

We assessed phosphorus metabolites in human parotid glands by ${ }^{31} \mathrm{P}-\mathrm{MRS}$ using 3D-CSI on 3T MRI. We evaluated whether this method could be used to capture changes in the levels of high-energy phosphorus metabolites (ATP and $\mathrm{PCr}$ ) due to saliva secretion.

\section{Materials and Methods}

\subsection{Ethical Approval of the Study Protocol}

The study was conducted in accordance with the ethical standards set by the World Medical Association (Declaration of Helsinki). Approval for the study protocol was obtained from the Institutional Review Board/Ethics Committee of the Kyoto University Graduate School of Medicine (R1382) on 22 February 
2018. Written informed consent was obtained from each participant prior to MRI.

\subsection{Participants}

Between March and July 2018, 20 healthy adult volunteers (12 men and 8 women; age range, 25 - 64 years; mean age, 36.8 years) were recruited through advertisements. The exclusion criteria were a history of salivary-gland disorders, communication difficulties, or contraindications to MRI (e.g., claustrophobia, metallic implants).

\subsection{MRS Examinations}

MRS data were acquired on 3T MRI (Magnetom Skyra; Siemens Healthcare, Erlangen, Germany), using a ${ }^{31} \mathrm{P} /{ }^{1} \mathrm{H}$ loop transmit-receive radiofrequency coil (Takashima Seisakusho, Tokyo, Japan) with a diameter of $120 \mathrm{~mm}$. Participants were placed in the right-lateral position in the magnet with the coil positioned over the lateral aspect of the right parotid gland. ${ }^{1} \mathrm{H}$ T1-weighted ultrafast gradient-echo images $(\mathrm{TR}=4.00 \mathrm{~ms}, \mathrm{TE}=0.99 \mathrm{~ms}$, FoV $=400 \mathrm{~mm}$, base resolution $=256$, slice thickness $=4.5 \mathrm{~mm}$, acquisition time $=48 \mathrm{~s}$ ) were obtained covering the right parotid gland, and these were used for voxel placement in ${ }^{31} \mathrm{P}$ spectroscopic imaging. $3 \mathrm{D}{ }^{31} \mathrm{P}$ spectroscopic images were obtained with the following parameters: $\mathrm{TR}=1000 \mathrm{~ms}, \mathrm{TE}=2.3 \mathrm{~ms}$, bandwidth $=3000 \mathrm{~Hz}, \mathrm{FoV}=200 \times 200$ $\times 200 \mathrm{~mm}$, matrix $=9 \times 9 \times 8$, and flip angle $=90^{\circ}$. The matrix size was interpolated to $16 \times 16 \times 16$, resulting in an interpolated voxel size of $2.2 \times 2.2 \times 2.5 \mathrm{~cm}$, or $12.3 \mathrm{~cm}^{3}$. Weighted averaging (12 averages) was applied, resulting in a total acquisition time of $10 \mathrm{~min}, 32 \mathrm{~s} .{ }^{31} \mathrm{P}$ MR spectra were acquired three times in all participants. As a baseline, the first measurement (pre) was obtained after $3 \mathrm{~h}$ of fasting. The subjects were then asked to take tablets containing $244 \mathrm{mg}$ of vitamin $C$, and measurements were obtained twice more, in a continuous manner (post-1 and post-2).

\subsection{Analyses of ${ }^{31} \mathrm{P}$ Data}

Analyses were undertaken by a single radiologist who had 7 years of experience. On the T1-weighted ultrafast gradient-echo images, a voxel in a homogeneous area (to avoid blood vessels) of the right parotid gland was chosen for evaluation. Spectra were analyzed with Syngo software (Syngo Spectroscopy Evaluation; Siemens Medical Systems). The intensities of phosphocholine, phosphoethanolamine, inorganic phosphate, glycerophosphoethanolamine, glycerophosphocholine, PCr, and ATP were fitted in the frequency domain using the least-squares method. Results were expressed as the ratio of the peak area to the total phosphorus signal.

\subsection{Statistical Analyses}

Results are expressed as the mean \pm standard deviation (SD). The $\beta$-ATP peak 
was used to estimate the ATP level because this peak does not contain adenosine monophosphate or ADP. Significant changes in the time-course of $\mathrm{PCr}$ and $\beta$-ATP were evaluated with one-way analysis of variance. Overall significant differences during the three-time points were further tested using the paired $t$-test with Bonferroni's correction. Statistical tests were two-sided, and $\mathrm{p}<0.05$ was considered significant. Statistical analyses were performed using R v3.2.4 (R Foundation for Statistical Computing, Vienna, Austria).

\section{Results}

Nine peaks were depicted in the ${ }^{31} \mathrm{P}$-MRS spectrum (Figure 1). Figure 2 shows

a

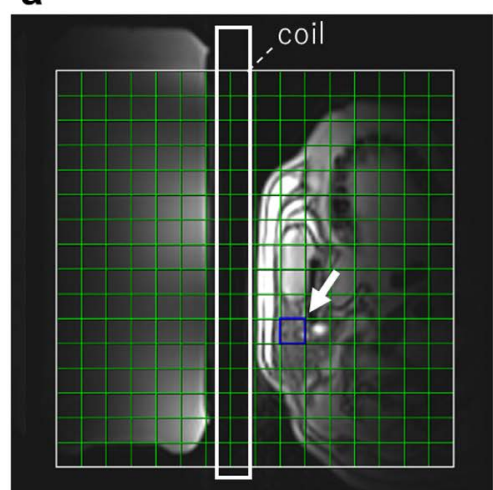

b

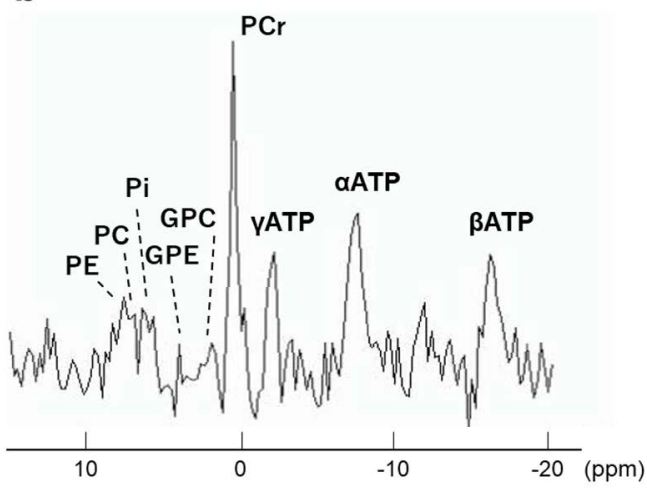

Figure 1. Axial MR image (a) of a parotid gland showing the selected voxel (arrow), and corresponding in vivo spectrum (b) acquired by 3D-CSI in $10 \mathrm{~min}, 32 \mathrm{~s}$. The assigned metabolite resonances are as follows: $\mathrm{PE}$, phosphoethanolamine; $\mathrm{PC}$, phosphocholine; $\mathrm{Pi}$, inorganic phosphate; GPE, glycerophosphoethanolamine; GPC, glycerophosphocholine; PCr, phosphocreatine; ATP, adenosine triphosphate.

0.4

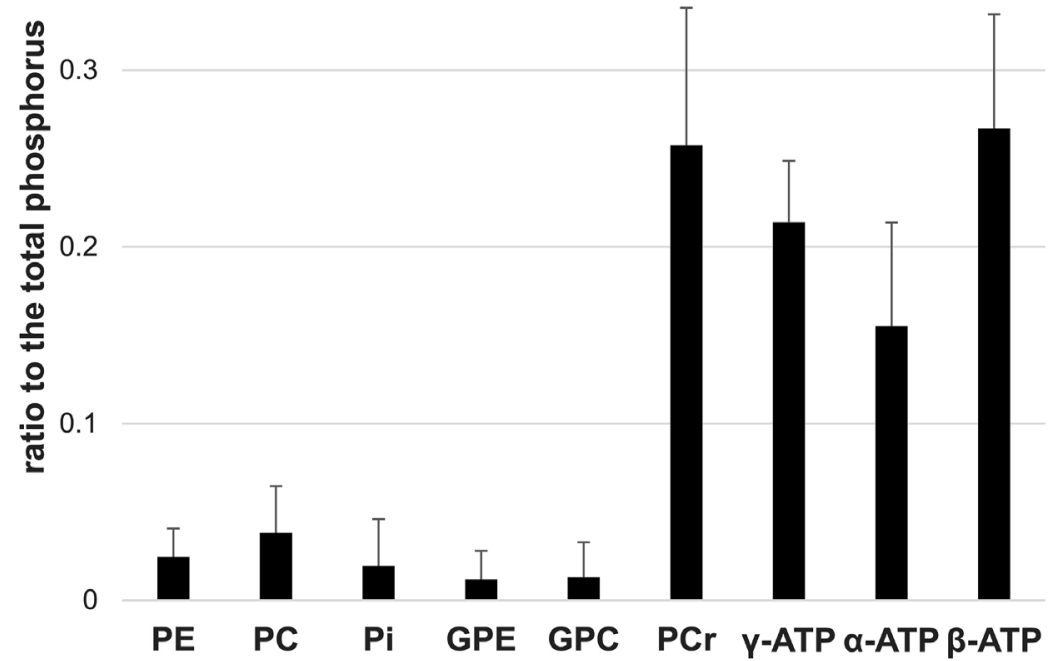

Figure 2. Levels of major phosphorus metabolites (mean $\pm \mathrm{SD}$ ) in the parotid glands of 20 healthy volunteers. 
the mean \pm SD of the ratio of each substance to the total phosphorus signal before vitamin-C stimulation in the 20 participants. The highest ratios were found for the high-energy phosphorus metabolites PCr $(0.26 \pm 0.08)$ and ATP $(\alpha: 0.16$ $\pm 0.06 ; \beta: 0.27 \pm 0.06 ; \gamma .0 .21 \pm 0.03)$. The signal-to-noise ratio of $\beta$-ATP and $\mathrm{PCr}$ was sufficient for detection and analysis ( $\beta$-ATP: $5.05 \pm 1.76$; PCr: $6.66 \pm 2.77$ ).

Figure 3 shows $\beta$-ATP and PCr levels before and after vitamin-C stimulation. A significant change was observed in the time-course of $\beta$-ATP $(\mathrm{p}<0.05)$. Multiple comparisons revealed a significant decrease between pre $(0.27 \pm 0.06)$ and post-1 $(0.19 \pm 0.07, \mathrm{p}<0.05)$, and there was a nearly significant increase between post-1 $(0.19 \pm 0.07)$ and post-2 $(0.26 \pm 0.08, \mathrm{p}=0.08)$. There was a significant change in the time-course of PCr $(\mathrm{p}<0.05)$ and a nearly significant decrease

\section{a}

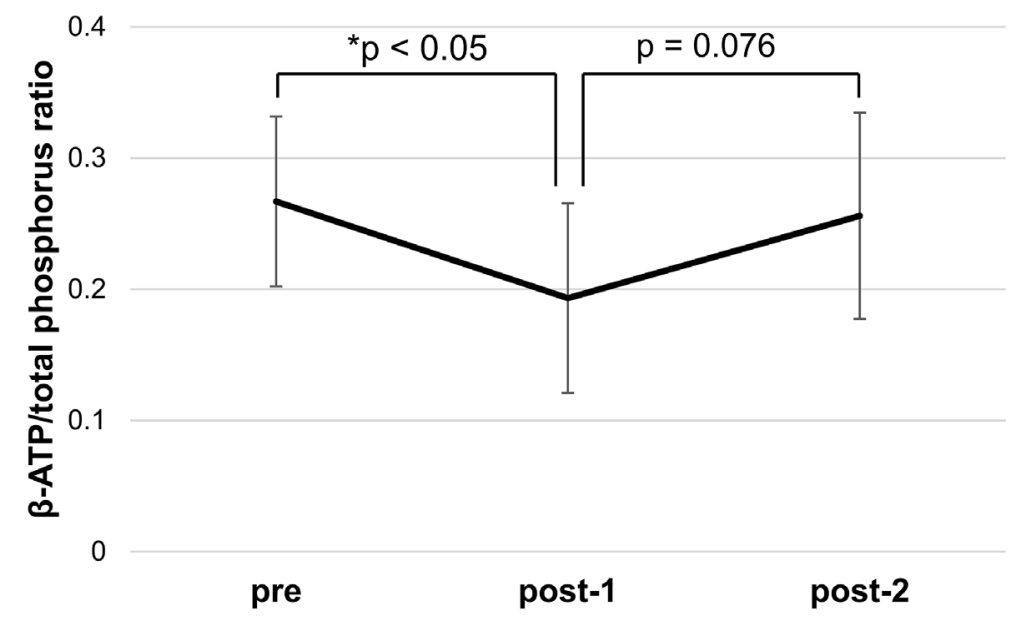

b

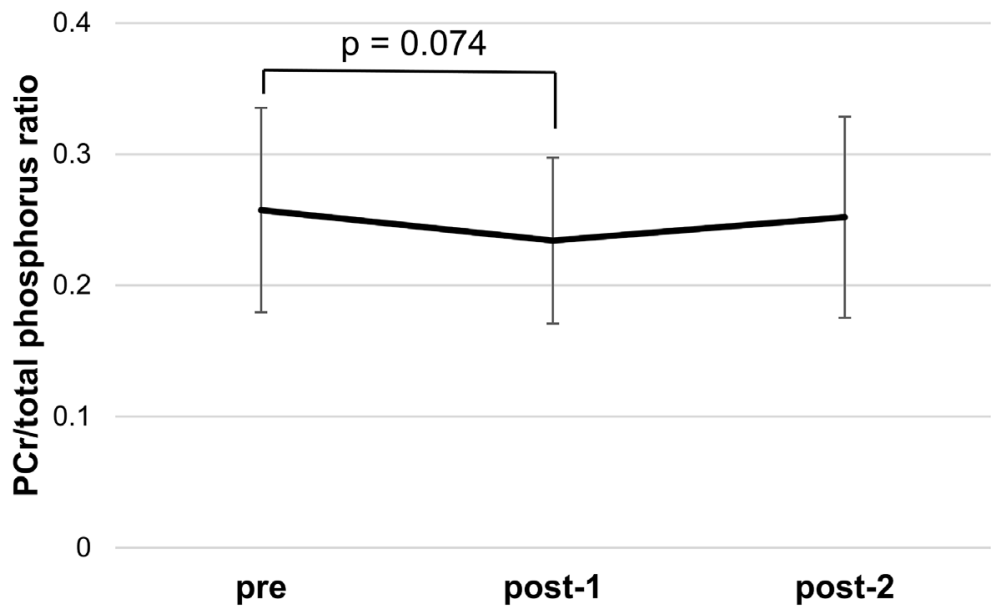

Figure 3. Levels of phosphorus metabolites (mean $\pm \mathrm{SD}$ ) at baseline and after vitamin-C stimulation. Ratio of the peak area to the total phosphorus signal for (a) $\beta$-adenosine triphosphate ( $\beta$-ATP) and (b) peak area ratio to the total phosphorus signal for phosphocreatine (PCr). ${ }^{\star}$ Denotes significant differences between groups at $\mathrm{p}<0.05$. 
between pre $(0.26 \pm 0.08)$ and post- $1(0.23 \pm 0.06, \mathrm{p}=0.07)$. There was a slight increase in the mean \pm SD between post- $1(0.23 \pm 0.06)$ and post- $2(0.25 \pm 0.08)$, but this change was not significant $(\mathrm{p}=1.00)$.

\section{Discussion}

Saliva secretion requires the coordinated activity of multiple water transporters, ion transporters, and ion-channel proteins, and phosphorus metabolism plays an important part. In the salivary glands, ATP is consumed by $\mathrm{Na}^{+} / \mathrm{K}^{+}$ATPase, which is believed to establish a $\mathrm{Na}^{+}$gradient across cell membranes to create the driving force for $\mathrm{Na}^{+}-\mathrm{K}^{+}-\mathrm{Cl}^{-}$cotransport systems. $\mathrm{Cl}^{-}$is then released to the lumen through calcium-activated apical $\mathrm{Cl}^{-}$channels. Following the movement of $\mathrm{Cl}^{-}, \mathrm{Na}^{+}$, and water into the lumen, saliva is secreted [17] [18] [19].

The aim of our study was to assess phosphorus metabolites in human parotid glands using ${ }^{31} \mathrm{P}$-MRS with $3 \mathrm{D}$-CSI on $3 \mathrm{~T}$ MRI, and additionally evaluate whether this technique could capture changes in phosphorus metabolites due to saliva secretion.

We detected a high proportion of the high-energy phosphorus metabolites PCr and ATP. ATP levels decreased significantly after vitamin-C stimulation, and PCr levels decreased to near-significant levels. These data suggest that, in vivo, ${ }^{31} \mathrm{P}$-MRS with $3 \mathrm{D}$-CSI can be used to detect major phosphorus metabolites in the human parotid gland within a reasonable scan time, and to reveal the consumption of high-energy phosphorus metabolites due to saliva secretion.

The spectra showed obvious peaks due to the high-energy phosphorus metabolites ATP and PCr. Our results are in accordance with reports from human and in vitro animal experiments [9]-[15]. Only one of these was a clinical study (15), which was performed using $1.5 \mathrm{~T}$ MRI, compared with $3 \mathrm{~T}$ MRI in the present study. In theory, a higher field strength should be advantageous for MRS because of its greater sensitivity and chemical-shift resolution, and shorter acquisition time [20] [21] [22]. The study that used 1.5T MRI [15] employed the surface-coil localization method and achieved only a static spectrum, with an acquisition time of $35 \mathrm{~min}$. Furthermore, no detailed information on position was obtained. However, in the present 3T MRI study, even though we employed 3D-CSI and obtained high spatial resolution, we could still achieve a shorter acquisition time (10 min, $32 \mathrm{~s}$ per scan) and continuous imaging. Also, we considered that the use of 3D-CSI would enable us to avoid the partial-volume effect and to reduce signal contamination, particularly that from muscles (which are rich in ATP and PCr).

A high signal intensity of PCr has been reported for the salivary glands, skeletal muscle, heart, and brain [4] [20] [21]. The Lohmann reaction (a reversible mechanism for maintaining the ATP concentration) exists in these organs, producing ATP by transferring high-energy phosphate groups from $\mathrm{PCr}$ to ADP. $\mathrm{PCr}$ is produced by the creatine kinase present in mitochondria, and it moves into cytoplasm with a high diffusion coefficient. On reaching the target ATPase, 
the high-energy phosphate group is transferred from $\mathrm{PCr}$ to ADP to produce ATP [22]. It is believed that PCr is abundant in organs that have a high demand for rapid energy. Our results indicate that physiological changes in the salivary glands require rapid liberation of energy.

We detected a decrease in the levels of high-energy phosphorus metabolites during saliva secretion upon vitamin-C stimulation. ${ }^{31} \mathrm{P}$-MRS has been used for three decades in studies of physiological changes in muscles, brain, and liver. However, studies of the salivary glands have been performed only with excised and perfused animal glands, and research on saliva production in humans is lacking. In animal experiments, reductions in PCr levels were more apparent than those of ATP [9]-[14]. In the present study, a significant decrease in ATP levels was observed after saliva secretion, whereas the decrease in PCr levels was nearly significant. Further work is needed to explore the reason for this result.

Methods for assessing the salivary glands include the gum method and the Saxon test to measure saliva secretion on stimulation, the spitting method to measure resting saliva secretion, and scintigraphy to evaluate function [23] [24]. Phosphorus metabolites can provide unique information other than the amount of saliva. It has been reported that static spectra of the salivary glands of patients with Sjögren's syndrome reveal low levels of ATP and PCr [15]; further information could be obtained using our method. In addition, by obtaining information regarding metabolism and position simultaneously with 3D-CSI, it may be possible to evaluate the function of each salivary gland.

Our study had three main limitations. First, we included only healthy volunteers in the study cohort. In future studies, it will be necessary to compare the results in healthy volunteers with those of patients with failure of salivary secretion. Second, we assessed the results in terms of the ratios of levels of metabolites. Although relative quantification is a convenient and useful way to capture trends, it is necessary to evaluate the absolute concentration to obtain more accurate and unambiguous results. Third, the resonances of phosphocholine, phosphoethanolamine, inorganic phosphate, glycerophosphoethanolamine, and glycerophosphocholine were lower than those stated previously [9]-[15] because we used a shorter TR to achieve a tolerable examination time. Nevertheless, this was not a serious problem in the present study because the main purpose was to evaluate changes in levels of the high-energy phosphorus metabolites ATP and PCr.

\section{Conclusion}

In conclusion, we demonstrated that 31P-MRS with 3D-CSI can be used to evaluate levels of the major phosphorus metabolites in human parotid glands, and to capture the change in levels of high-energy phosphate groups due to salivary secretion. Acquisition time was $10 \mathrm{~min}, 32 \mathrm{~s}$ per image, and it was possible to acquire images continuously. The examination was not burdensome for the study participants. Our technique could be applied in much larger studies to 
evaluate the pathogenesis of saliva-secretion failure (e.g., Sjögren's syndrome and radiation-induced salivary gland dysfunction).

\section{Acknowledgements}

The authors thank Ryutaro Nakagami (Siemens Healthcare K.K.) for technical assistance with experiments. We thank Forte Science Communications (Tokyo, Japan) for editorial assistance with the manuscript.

\section{Funding}

This research did not receive any specific grant from funding agencies in the public, commercial, or not-for-profit sectors.

\section{Conflicts of Interest}

The authors declare no conflicts of interest regarding the publication of this paper.

\section{References}

[1] Dawes, C. and Wood, C.M. (1973) The Contribution of Oral Minor Mucous Gland Secretions to the Volume of Whole Saliva in Man. Archives of Oral Biology, 18, 337-342. https://doi.org/10.1016/0003-9969(73)90156-8

[2] Qi, J., Shukla-Dave, A., Fong, Y., Gönen, M., Schwartz, L.H., Jarnagin, W.M., et al. (2011) 31P MR Spectroscopic Imaging Detects Regenerative Changes in Human Liver Stimulated by Portal Vein Embolization. Journal of Magnetic Resonance Imaging, 34, 336-344. https://doi.org/10.1002/jmri.22616

[3] Lin, Y.-C., Wu, J., Baltzis, D., Veves, A. and Greenman, R. (2016) MRI Assessment of Regional Differences in Phosphorus-31 Metabolism and Morphological Abnormalities of the Foot Muscles in Diabetes. Journal of Magnetic Resonance Imaging, 44, 1132-1142. https://doi.org/10.1002/jmri.25278

[4] Chance, B., Eleff, S., Leigh, J.S., Sokolow, D. and Sapega, A. (1981) Mitochondrial Regulation of Phosphocreatine/Inorganic Phosphate Ratios in Exercising Human Muscle: A Gated 31P NMR Study. Proceedings of the National Academy of Sciences, 78, 6714-6718. https://doi.org/10.1073/pnas.78.11.6714

[5] Boesch, C., Elsing, C., Wegmuller, H., Felblinger, J., Vock, P. and Reichen, J. (1997) Effect of Ethanol and Fructose on Liver Metabolism: A Dynamic 31Phosphorus Magnetic Resonance Spectroscopy Study in Normal Volunteers. Magnetic Resonance Imaging, 15, 1067-1077. https://doi.org/10.1016/S0730-725X(97)00163-X

[6] Kato, T., Murashita, J., Shioiri, T., Hamakawa, H. and Inubushi, T. (1996) Effect of Photic Stimulation on Energy Metabolism in the Human Brain Measured by 31P-MR Spectroscopy. The Journal of Neuropsychiatry and Clinical Neurosciences, 8, 417-422. https://doi.org/10.1176/jnp.8.4.417

[7] Chmelík, M., Schmid, A.I., Gruber, S., Szendroedi, J., Krššák, M., Trattnig, S., et al. (2008) Three-Dimensional High-Resolution Magnetic Resonance Spectroscopic Imaging for Absolute Quantification of 31P Metabolites in Human Liver. Magnetic Resonance in Medicine, 60, 796-802. https://doi.org/10.1002/mrm.21762

[8] Szendroedi, J., Chmelik, M., Schmid, A.I., Nowotny, P., Brehm, A., Krssak, M., et al. 
(2009) Abnormal Hepatic Energy Homeostasis in Type 2 Diabetes. Hepatology, 50, 1079-1086. https://doi.org/10.1002/hep.23093

[9] Murakami, M., Imai, Y., Seo, Y., Morimoto, T., Shiga, K. and Watari, H. (1983) Phosphorus Nuclear Magnetic Resonance of Perfused Salivary Gland. Biochimica et Biophysica Acta, 762, 19-24. https://doi.org/10.1016/0167-4889(83)90111-8

[10] Nakahari, T., Seo, Y., Murakami, M., Mori, H., Miyamoto, S., Imai, Y., et al. (1985) 31P-NMR Study of Dog Submandibular Gland in Vivo and in Vitro Using the Topical Magnetic Resonance. The Japanese Journal of Physiology, 35, 729-240. https://doi.org/10.2170/jiphysiol.35.729

[11] Murakami, M., Seo, Y., Watari, H., Ueda, H., Hashimoto, T. and Tagawa, K. (1987) 31P NMR Studies on the Isolated Perfused Mandibular Gland of the Rat. The Japanese Journal of Physiology, 37, 411-423.

https://doi.org/10.2170/jjphysiol.37.411

[12] Murakami, M., Seo, Y. and Watari, H. (1988) Dissociation of Fluid Secretion and Energy Supply in Rat Mandibular Gland by High Dose of ACh. The American Journal of Physiology, 254, G781-G787. https://doi.org/10.1152/ajpgi.1988.254.5.G781

[13] Seo, Y., Steward, M.C., Mackenzie, I.S. and Case, R.M. (1988) Acetylcholine-Induced Metabolic Changes in the Perfused Rabbit Mandibular Salivary Gland Studied by 31P-NMR Spectroscopy. Biochimica et Biophysica Acta, 971, 289-297. https://doi.org/10.1016/0167-4889(88)90144-9

[14] Steward, M.C., Seo, Y. and Case, R.M. (1989) Intracellular pH during Secretion in the Perfused Rabbit Mandibular Salivary Gland Measured by 31P NMR Spectroscopy. Pflugers Archiv: European Journal of Physiology, 414, 200-2007. https://doi.org/10.1007/BF00580964

[15] Vogl, T.J., Dadashi, A., Jassoy, A., Becker, C., Reimann, V. and Lissner, J. (1993) [The 31-Phosphorus Spectroscopy of Space-Occupying Lesions of the Salivary Glands. The Clinical Results and Differential Diagnosis]. RoFo: Fortschritte auf dem Gebiete der Rontgenstrahlen und der Nuklearmedizin, 158, 31-38. https://doi.org/10.1055/s-2008-1032596

[16] Furuyama, S., Abe, M., Yokoyama, N., Sugiya, H. and Fujita, Y. (1980) Mitochondrial Creatine Kinase in Rat Submandibular Gland. The International Journal of Biochemistry, 11, 259-264. https://doi.org/10.1016/0020-711X(80)90228-1

[17] Turner, R.J. and Sugiya, H. (2002) Understanding Salivary Fluid and Protein Secretion. Oral Diseases, 8, 3-11. https://doi.org/10.1034/j.1601-0825.2002.10815.x

[18] Melvin, J.E., Moran, A. and Turner, R.J. (1988) The Role of $\mathrm{HCO}_{3}^{-}$and $\mathrm{Na}+\mathrm{H}+$ Exchange in the Response of Rat Parotid Acinar Cells to Muscarinic Stimulation. The Journal of Biological Chemistry, 263, 19564-19569.

[19] Turner, R.J. and George, J.N. (1988) $\mathrm{Cl}^{-}-\mathrm{HCO}_{3}^{-}$Exchange Is Present with $\mathrm{Na}^{+}-\mathrm{K}^{+}-\mathrm{Cl}^{-}$Cotransport in Rabbit Parotid Acinar Basolateral Membranes. The American Journal of Physiology, 254, C391-C396. https://doi.org/10.1152/ajpcell.1988.254.3.C391

[20] Bottomley, P.A. (1994) MR Spectroscopy of the Human Heart: The Status and the Challenges. Radiology, 191, 593-612. https://doi.org/10.1148/radiology.191.3.8184033

[21] Glonek, T., Kopp, S.J., Kot, E., Pettegrew, J.W., Harrison, W.H. and Cohen, M.M. (1982) P-31 Nuclear Magnetic Resonance Analysis of Brain: The Perchloric Acid Extract Spectrum. Journal of Neurochemistry, 39, 1210-1219. https://doi.org/10.1111/j.1471-4159.1982.tb12557.x 
[22] Savabi, F., Geiger, P.J. and Bessman, S.P. (1984) Myofibrillar End of the Creatine Phosphate Energy Shuttle. The American Journal of Physiology, 247, C424-C432. https://doi.org/10.1152/ajpcell.1984.247.5.C424

[23] Hochberg, M.C., Tielsch, J., Munoz, B., Bandeen-Roche, K., West, S.K. and Schein, O.D. (1998) Prevalence of Symptoms of Dry Mouth and Their Relationship to Saliva Production in Community Dwelling Elderly: The SEE Project. Salisbury Eye Evaluation. The Journal of Rheumatology, 25, 486-491.

[24] Navazesh, M. and Kumar, S.K. (2008) Measuring Salivary Flow: Challenges and Opportunities. Journal of the American Dental Association, 139, 35s-40s.

https://doi.org/10.14219/jada.archive.2008.0353 